\title{
Urinary Periostin Excretion Predicts Renal Outcome in IgA Nephropathy
}

\author{
Jin Ho Hwang ${ }^{a}$ Jung Pyo Lee ${ }^{b}$ Clara Tammy Kim ${ }^{c}$ Seung Hee Yang ${ }^{d}$ \\ Jin Hyuk Kim ${ }^{b}$ Jung Nam An ${ }^{b}$ Kyung Chul Moon ${ }^{e}$ Hajeong Lee ${ }^{f}$ \\ Yun Kyu Ohb Kwon Wook Joo ${ }^{\mathrm{b}}$ Dong Ki Kim ${ }^{\mathrm{f}}$ Yon Su Kim ${ }^{\mathrm{f}}$ Chun Soo Limb, \\ ${ }^{a}$ Department of Internal Medicine, Chung-Ang University Hospital, ${ }^{b}$ Department of Internal Medicine, Seoul \\ National University Boramae Medical Center, ${ }^{\mathrm{C}}$ Graduate School of Public Health, Seoul National University, ${ }^{\mathrm{d}}$ Kidney \\ Research Institute, Seoul National University Hospital, ${ }^{\circ}$ Department of Pathology, and ${ }^{\mathrm{f}}$ Department of Internal \\ Medicine, Seoul National University College of Medicine, Seoul, South Korea
}

\section{Key Words}

Biomarker · Fibrosis · IgA nephropathy · Periostin · Urine

\begin{abstract}
Background: Periostin is a matricellular protein and plays a vital role in tissue regeneration, fibrosis and wound healing. However, data about its significance in nephrology are limited. We investigated the correlation between urinary periostin excretion and its clinical significance including renal histologic findings and prognosis in IgA nephropathy (IgAN). Methods: Of 399 patients from a glomerulonephritis cohort recruited between January 2009 and December 2014, 314 were enrolled. Serum and urine periostin (UPOSTN) were measured using enzyme-linked immunosorbent assay. We divided the patients into 3 groups by uPOSTN/creatinine (uPOSTN/Cr): group 1 (undetectable), group 2 (lower than the median) and group 3 (higher than the median). Results: The uPOSTN level was correlated with pathologic classifications and both initial and final IDMS-MDRD estimated glomerular filtration rates (eGFRs; $p<0.001$ ). Histologically, group 3 patients were correlated with severe interstitial fibrosis/tubular atrophy $(p=0.004)$, interstitial inflammation $(p=0.007)$, hyaline arteriolosclerosis $(p=0.001)$ and glomerular sclerosis $(p<0.001)$. A higher initial uPOSTN/Cr level was
\end{abstract}

associated with a greater decline in eGFR during follow-up ( $p=0.043$ when initial eGFR $\geq 60 ; p=0.025$ when eGFR $<60$ $\left.\mathrm{ml} / \mathrm{min} / 1.73 \mathrm{~m}^{2}\right)$, and the renal outcomes with end-stage renal disease (ESRD; $p=0.003), E S R D$ and/or eGFR decrease of $>30 \%(p=0.033)$ and ESRD and/or eGFR decrease of $>50 \%$ ( $p=0.046$ ) occurred significantly more in group 3 . In multivariate analysis, uPOSTN group 3 (hazards ratio 2.839, 95\% Cl 1.013-7.957; $p=0.047$ ) was independently associated with ESRD in IgAN patients. Conclusion: $\mathrm{UPOSTN} / \mathrm{Cr}$ value at initial diagnosis correlated with renal fibrosis and predicted the renal outcomes in patients with IgAN. It could be a promising urinary biomarker for renal fibrosis.

(c) 2016 S. Karger AG, Basel

\section{Introduction}

Chronic kidney disease (CKD) is increasing the burden of public health worldwide. Various chronic diseases such as diabetes mellitus (DM), hypertension and glomerulonephritis cause CKD; yet, the common irreversible pathologic outcome is renal fibrosis [1].

J.H.H. and J.P.L. contributed equally to this research.

\section{KARGER}

E-Mail karger@karger.com

www.karger.com/ajn
(C) 2016 S. Karger AG, Basel

0250-8095/16/0446-0481\$39.50/0
Chun Soo Lim, MD, PhD

Department of Internal Medicine, Seoul National University College of Medicine Seoul National University Boramae Medical Center

20 Boramae-ro 5-gil, Dongjak-gu, Seoul 156-707 (South Korea)

E-Mail cslimjy@snu.ac.kr 
$\operatorname{Ig} \mathrm{A}$ nephropathy (IgAN) is one of the most common forms of glomerulonephritis and a frequent cause of endstage renal disease (ESRD) worldwide [2]. In Korea, it accounts for approximately half of all primary glomerulonephritis [3]. IgAN represents various clinical features, from lifelong mild hematuria with or without proteinuria to rapid decline or loss of renal function after diagnosis. The prognosis of IgAN is unclear because of the lack of renal biopsy data on suspicious cases of IgAN with a clinically mild disease course and the low accuracy of diagnosis [4]. In one study on biopsy-proven IgAN population in Korea, ESRD occurred in $20.6 \%$ of patients and the median time to ESRD was 71 months after renal biopsy [5]. Owing to the heterogeneity of reports of disease course and clinical features of IgAN, reliably predicting the outcome at diagnosis is difficult. Therefore, the development of noninvasive biomarkers with high sensitivity and specificity that can be used for predicting disease prognosis would be highly beneficial. Urinary biomarkers have several advantages; they could be collected in large volumes using noninvasive methods, and they are stable owing to the lack of endogenous proteases [6].

Periostin is a nonstructural matricellular protein that plays a major role in tissue regeneration, fibrosis and wound healing $[7,8]$. Reportedly, high periostin expression is related to asthma, heart failure, myocardial infarction and various forms of human cancers [9-12]. Periostin is induced during nephrogenesis, but is not found in a normal adult kidney [13]. In the field of nephrology, periostin-related research is emerging. Periostin is mainly expressed in the tubulointerstitial areas in renal fibrosis, and studies have shown a relationship between urinary periostin level and tubular damage, CKD (in an experimental animal model), autosomal dominant polycystic kidney disease and chronic allograft nephropathy [14-17]. Recent research has elucidated the significance of periostin as tissue or urinary biomarker in type $2 \mathrm{DM}$ and lupus nephritis $[18,19]$.

There was no information on the relationship between urinary periostin and clinical and pathological IgAN findings. The aim of this study was to investigate the correlation between urinary periostin excretion and its clinical significance, including renal histologic findings in IgAN.

\section{Methods}

\section{Study Design and Population}

We included patients who underwent kidney biopsy at Seoul National University Hospital and Seoul National University Boramae Medical Center. The prospective cohort studies were conducted in these 2 centers, and they manage biobanks for urine, plasma, serum, genomic DNA and biopsied tissue of all patients who underwent renal biopsy and provided informed consent.

We collected data of 399 adult patients with biopsy-proven IgAN from January 2009 to December 2014. We excluded 54 patients owing to non-availability of urine or plasma samples, insufficient follow-up or clinical data within 3 months of kidney biopsy (52 patients) and misdiagnosis (2 patients). Finally, 345 IgAN patients were included in the study. In addition, 56 patients were randomly selected from the Seoul National University Boramae Medical Center Human Biobank for comparison with IgAN patients.

Data about clinical parameters like age, sex, body mass index, blood chemistry, spot urine protein-creatinine ratio (uPCr) and comorbidity (hypertension, DM and viral hepatitis) were collected at the time of kidney biopsy. Information on blood chemistry, $\mathrm{uPCr}$ and prescribed medications (angiotensin-converting enzyme inhibitors or angiotensin II receptor blockers and immunosuppressive agents) was recorded during the follow-up period. Estimated glomerular filtration rate (eGFR) was calculated using the isotope dilution mass spectrometry - traceable modified Modification of Diet in Renal Disease equation. Biopsy tissues were examined using light, electron and immunofluorescence microscopy. The histologic diagnosis was made by a renal pathologist. Interstitial fibrosis and tubular atrophy (IF/TA) and interstitial inflammation scores were graded as a percentage of affected area as follows: 0 (none), 1 (mild, $\leq 25 \%$ ), 2 (moderate, $26-50 \%$ ) and 3 (severe, $>50 \%$ ). Information on fibrointimal thickening and hyaline arteriolosclerosis was obtained for the final pathologic record of each patient. To ensure accuracy of histopathologic findings, 2 independent pathologists reviewed 30 random samples each, which were re-evaluated in a blind manner.

This study was approved by the Institutional Review Board (H-1308-120-517), and the patients provided informed consent prior to recruitment into the cohort. All clinical investigations were conducted in accordance with the guidelines of the 2013 Declaration of Helsinki.

\section{Measurement of Urine and Plasma Periostin Levels}

Urine and plasma periostin levels were measured using enzyme-linked immunosorbent assay (ELISA; R\&D systems, Minneapolis, Minn., USA) according to the manufacturer-provided protocol. Ninety-six-well microplates were coated with $1 \mu \mathrm{g} / \mathrm{ml}$ $(0.1 \mu \mathrm{g}, 100 \mu \mathrm{l}$ per well) of anti-periostin antibody (R\&D systems) and incubated overnight. Plates were washed 3 times with $0.05 \%$ Tween-20 in phosphate-buffered saline and blocked with reagent diluent for more than $1 \mathrm{~h} ; 100 \mu \mathrm{l}$ of all standards and patient samples were added in each well and incubated for $2 \mathrm{~h}$ at room temperature. After 2-hour incubation with detection antibody, it was incubated for $20 \mathrm{~min}$ with dextran polymer conjugated with horseradish peroxidase and for 20 min with substrate solution, after which the stop solution was added to each well. Periostin absorbance was calculated by measuring at $450 \mathrm{~nm}$, correcting for plate artifact at $570 \mathrm{~nm}$ and using a 4-parameter logistic standard curve. Measured urine periostin (uPOSTN) levels were adjusted by urine creatinine. All measurements were performed in duplicate and in a blind manner. Urine creatinine concentrations were measured in the same urine samples and expressed as the uPOSTN/creatinine ratio (uPOSTN/Cr). Plasma samples were diluted at 1:50 before sample loading. 
Grouping and Outcomes

We divided the patients into 3 groups by uPOSTN/Cr: group 1 (undetectable level of uPOSTN), group 2 (below median value of measured uPOSTN/Cr) and group 3 (above median value of measured uPOSTN/Cr). We also evaluated the patients with plasma POSTN by quartile groups.

Renal outcomes were compared between the low uPOSTN group (groups 1 and 2) and group 3. The occurrence of ESRD was the primary outcome in this study. An eGFR decrease $\geq 30 \%$ and/ or ESRD, and eGFR decrease $\geq 50 \%$ and/or ESRD were secondary outcomes. The change in eGFR per year $(\triangle \mathrm{eGFR} /$ year $)$ was also evaluated by collecting final follow-up data and assessing the duration of total follow-up. We evaluated the association between uPOSTN/Cr and pathological parameters.

\section{Immunohistochemistry of Biopsy Slides}

Every obtainable, unstained slide from the study population was used for periostin immunostaining. Four-micron sections of formalin-fixed paraffin-embedded tissue were deparaffinized and rehydrated using xylene and ethanol. Endogenous streptavidin activity was blocked using $3 \%$ hydrogen peroxide. To examine the expression of human periostin, staining was performed using polyclonal periostin antibody (1:300; Abcam, Cambridge, Mass., USA) at $4^{\circ} \mathrm{C}$ overnight incubation, followed by incubation with dextran polymer conjugated with horseradish peroxidase (DAKO, Carpintería, Calif., USA) for $30 \mathrm{~min}$ at room temperature. For each kidney sample, 8 fields were viewed at $100 \times$ magnification under a light microscope and evaluated the periostin positivity area (\%) by morphometry system (Qwin 3, Leica, Netherlands). All slides were assessed by a single pathologist who was blind about the patients' pathologic findings or uPOSTN/Cr level.

\section{Cell Culture and Analysis of mRNA Expression}

Human collecting duct cells ( $\mathrm{H} 5$ cell line) were cultured at $37^{\circ} \mathrm{C}$ in a $5 \%$ carbon dioxide atmosphere in Dulbecco's Modified Eagle's Medium/F-12 1:1 mixture with HEPES, L-glutamine (Lonza, Basel, Switzerland) supplemented with $10 \%$ fetal bovine serum, $5 \mathrm{mg} / \mathrm{ml}$ apotransferrin, 3.10-4 $\mathrm{M}$ sodium selenite, $10 \mathrm{mg} / \mathrm{ml}$ insulin from bovine pancreas and 10-3 $\mathrm{M}$ dexamethasone (cell line and protocol were provided by Remi Piedagnel). Cells were grown to approximately $80 \%$ confluence and subjected to serum deprivation (1\% FBS) for $24 \mathrm{~h}$ before experimental cell treatment. The $\mathrm{H} 5$ cells were treated with $10 \mathrm{ng}$ TGF- $\beta$ (positive control) or $0.25,0.5$ or $1.0 \mu$ g periostin for $24 \mathrm{~h}$. The TGF- $\beta$ and periostin were purchased from R\&D systems. Total RNA was isolated from cells using the RNeasy ${ }^{\circledR}$ kit (Qiagen $\mathrm{GmBH}$, Hilden, Germany), and $1 \mu \mathrm{g}$ of total RNA was reverse transcribed using oligo-d(T) primers and AMV-RT Taq polymerase (Promega, Madison, Wis., USA). Real-time polymerase chain reaction was performed using assay-on-demand TaqMan ${ }^{\circledR}$ probes and primers for type I, alpha 1 collagen (collagen Ia 1) and glyceraldehyde 3-phosphate dehydrogenase (GAPDH; Applied Biosystems) and an ABI PRISM ${ }^{\circledR} 7500$ Sequence Detection System (Applied Biosystems). The levels of mRNA expression for collagen Ia1 were normalized with respect to the level of GAPDH mRNA expression.

\section{Statistical Analysis}

Continuous variables were expressed as mean $\pm \mathrm{SD}$, and categorical variables as the number and percentage. The independent samples $t$ test was used for continuous variables. For multiple comparisons, one-way analysis of variance was used followed by the least significant difference test. Categorical data were compared using the chi-square test. Pearson correlation coefficients were determined to explore the relationship between uPOSTN and plasma periostin, initial eGFR and uPCr. The ESRD-free survival rates and other event-free survival rates were calculated using the KaplanMeier method, and comparison between groups was performed using the log-rank test. The Cox regression model was used to identify independent risk factors for ESRD, and to calculate the hazards ratio (HR) and 95\% CI. Finally, receiver operating characteristics (ROC) analysis was used to calculate the area under the curve (AUC) for uPOSTN/Cr level and to find the best cutoff value to predict the outcome of ESRD. To assess and compare the predictive performance of uPOSTN/Cr, time-dependent ROC curves for censored survival data and AUC at 1,2 and 3 years after diagnosis were additionally used. Most analyses and calculations were performed using the IBM SPSS Statistics version 21.0 (IBM Corporation, Armonk, N.Y., USA). We performed ROC analysis using R version 3.2.0 (R Foundation for Statistical Computing, Wien, Austria). Statistical significance was set at the level of $\mathrm{p}<0.05$.

\section{Results}

\section{Baseline Characteristics by Urine and Plasma POSTN Grouping}

The study flow chart is shown in online supplementary figure 1 (for all online suppl. material, see www.karger.com/doi/10.1159/000452228). The distribution of plasma periostin, the uPOSTN/Cr levels of healthy subjects and of IgAN patients and the natural logarithm transformed (ln) uPOSTN/Cr level of these patients are shown in figure la-c, respectively. Table 1 summarizes baseline characteristics of the study populations by uPOSTN/Cr level; 148 patients who had undetected uPOSTN level by ELISA were categorized into group 1 . Remaining patients with uPOSTN levels measured by ELISA were divided into groups 2 and 3 by the median value (median uPOSTN/Cr, $628.1 \mathrm{pg} / \mathrm{mgCr}$ ). Systolic and diastolic blood pressures were higher, and eGFR at the time of renal biopsy was lower in group 3. Pathologic classifications were compared between groups, and patients with advanced grades or classes were distributed significantly more in group 3 (table 1). Total follow-up duration was 27.1 months, and that of groups 1, 2 and 3 was 20.9, 29.1 and 32.9 months, respectively $(\mathrm{p}<0.001)$.

The quartile groups by plasma periostin level did not show significant differences in most of the parameters between groups (online suppl. table S1). In correlation analysis, uPOSTN/Cr was weakly associated with plasma POSTN ( $r=0.232 ; \mathrm{p}=0.007$; fig. 1d). Urinary POSTN/Cr showed negative correlation with eGFR at the time of renal biopsy ( $r=-0.245 ; \mathrm{p}=0.001$; fig. $1 \mathrm{e}$ ) and showed no significant relationship with uPCr (fig. 1f). 


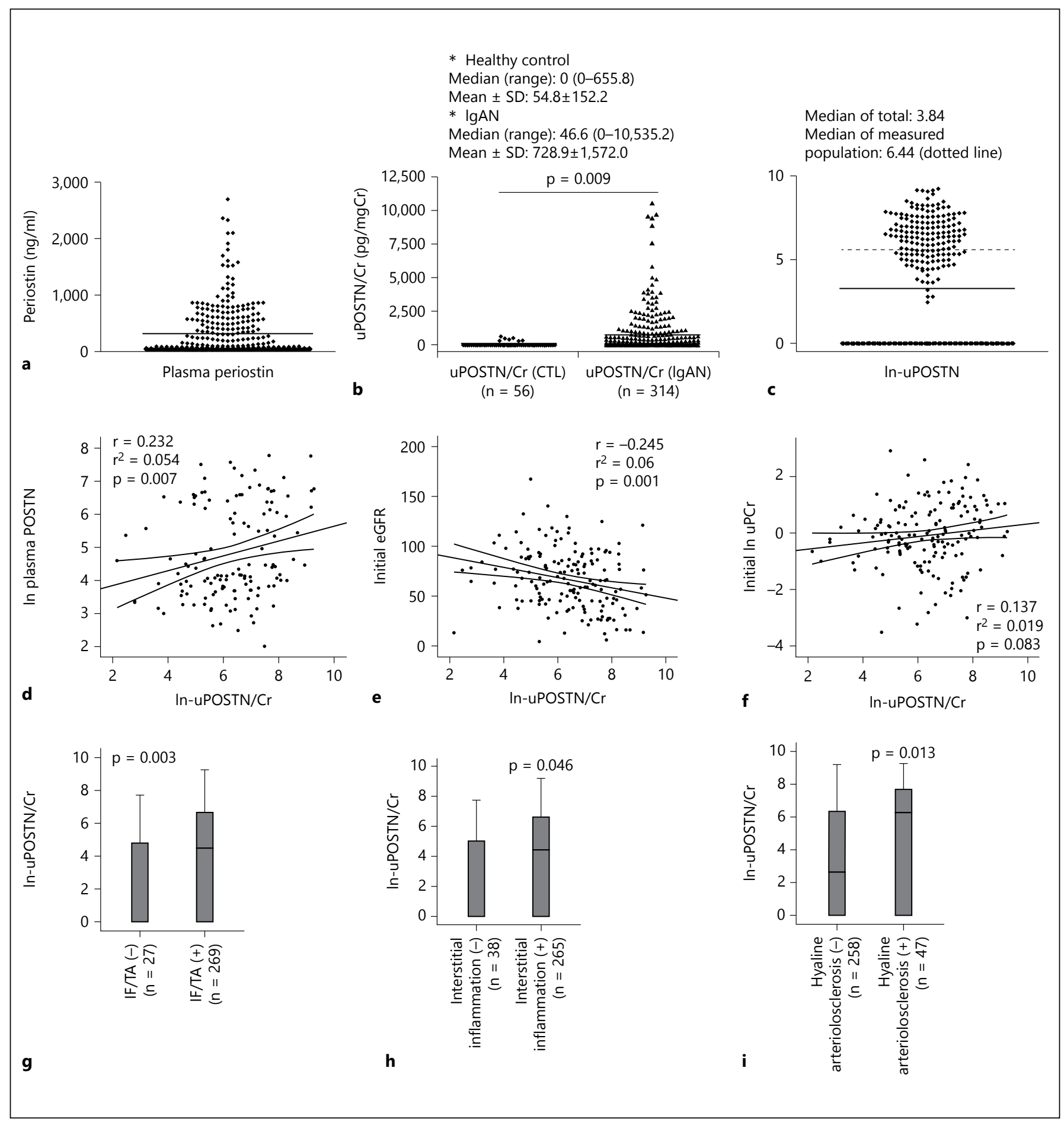

Fig. 1. Relationships between uPOSTN/Cr and clinical parameters. Distribution of (a) plasma periostin $(\mathrm{ng} / \mathrm{ml})$ and (b) uPOSTN/Cr in healthy control (CTL) compared with patients with IgAN and (c) natural $\log$ transformed (ln-)levels of uPOSTN/Cr. Correlation between (d) uPOSTN/Cr and plasma
POSTN $(\mathrm{r}=0.232 ; \mathrm{p}=0.007),(\mathbf{e}) \mathrm{uPOSTN} / \mathrm{Cr}$ and initial eGFR $(\mathrm{r}=-0.245 ; \mathrm{p}=0.001)$, and $(\mathbf{f}) \mathrm{uPOSTN} / \mathrm{Cr}$ and initial $\mathrm{uPCR}(\mathrm{r}=$ $0.137 ; \mathrm{p}=0.083)$. Ln-uPOSTN/Cr level $(\mathbf{g})$ with or without IF/ TA $(\mathrm{p}=0.003),(\mathbf{h})$ by interstitial inflammation $(\mathrm{p}=0.046)$ and (i) by hyaline arteriolosclerosis $(\mathrm{p}=0.013)$. 
Table 1. Baseline characteristics of the study subjects

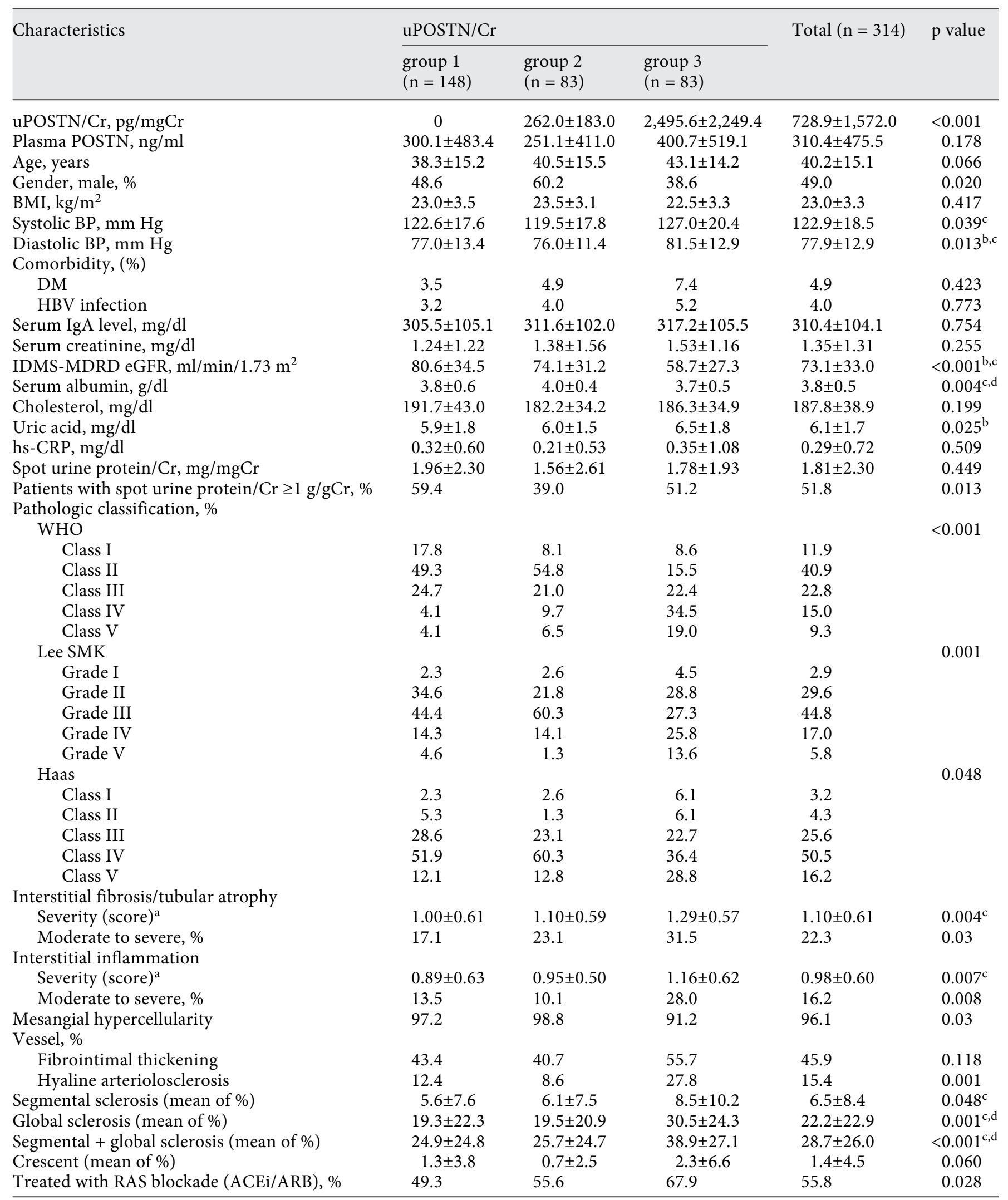

uPOSTN/Cr and Renal Outcome in IgAN 
Table 1. (continued)

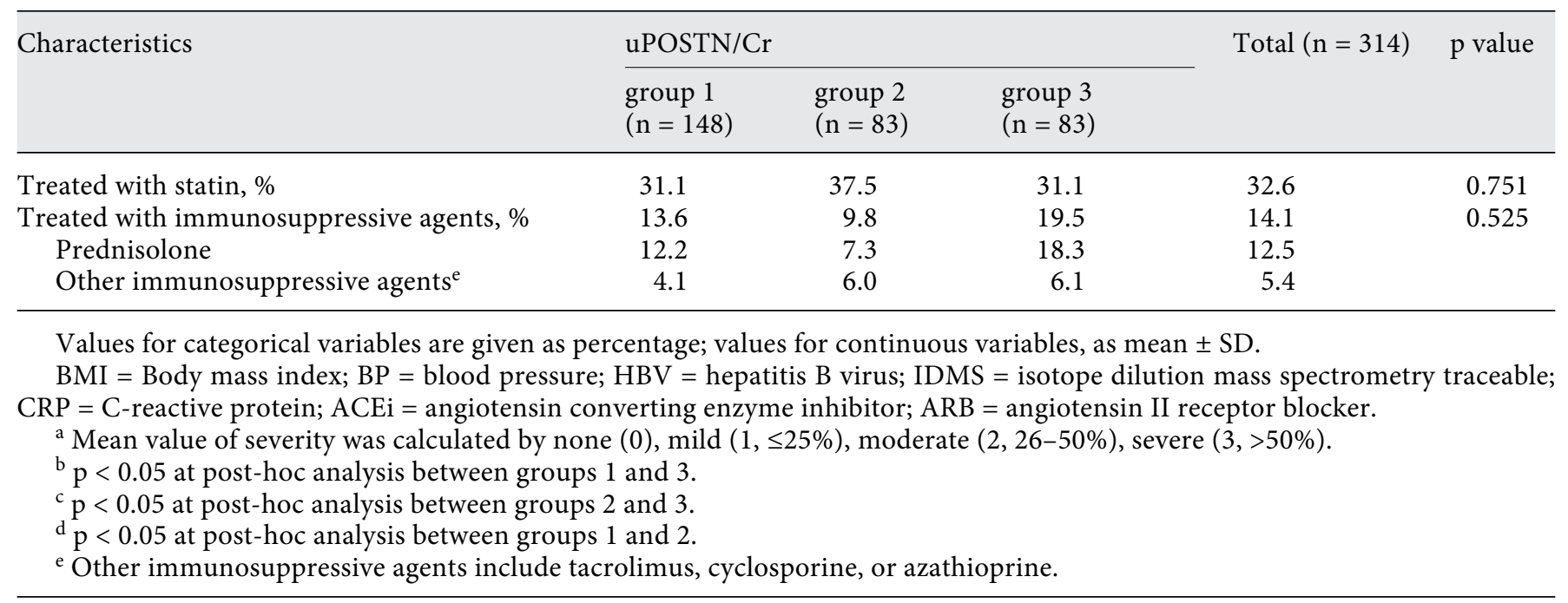

uPOSTN/Cr and Histologic Findings

Figure 1g-i shows the ln-uPOSTN/Cr levels in significant pathologic findings. The patients with IF/TA ( $\mathrm{p}=$ 0.003; fig. $1 \mathrm{~g}$ ), interstitial inflammation ( $\mathrm{p}=0.046$; fig. $1 \mathrm{~h}$ ) and hyaline arteriolosclerosis $(\mathrm{p}=0.013$; fig. 1i) showed higher ln-uPOSTN/Cr levels than the others.

\section{Renal Outcomes}

ESRD developed in 28 patients during the mean 27.1 months of follow-up. We evaluated the renal outcomes between group $1+2$ and group 3 because baseline characteristics were significantly different, particularly in group 3. All the renal outcomes with ESRD $(p=0.003)$, ESRD and/or eGFR decrease $>30 \%(\mathrm{p}=0.033)$ and ESRD and/or eGFR decrease $>50 \%(\mathrm{p}=0.046)$ occurred significantly more in group 3 as per Kaplan-Meier survival analyses (fig. 2a-c).

The patients with ESRD $(\mathrm{p}<0.001)$, eGFR decrease $>30 \%(p=0.002)$ and eGFR decrease $>50 \%(p=$ 0.046 ) showed higher $\ln$-uPOSTN/Cr levels than patients without these renal outcomes (fig. 2d-f).

We determined Pearson correlation coefficients between various clinical parameters (online suppl. fig. S2) and conducted multivariate analysis for the occurrence of ESRD. When we adjusted for age, sex, uPCr and serum creatinine at the time of renal biopsy, and uPOSTN-based grouping, we found that $\mathrm{uPCr}(\mathrm{p}<0.001)$, serum creatinine $(\mathrm{p}<0.001)$ and uPOSTN group 3 (HR 2.839 vs. group $1+2 ; 95 \%$ CI $1.013-7.957 ; \mathrm{p}=0.047$ ) independently associated with ESRD in IgAN patients (table 2).
We compared annual eGFR decrease ( $\triangle \mathrm{eGFR} / \mathrm{year})$ only in patients followed up for more than 1 year. The patients in group 3 (high uPOSTN/Cr), those with initial eGFR $\geq 60 \mathrm{ml} / \mathrm{min} / 1.73 \mathrm{~m}^{2}(\mathrm{p}=0.043)$ and $<60 \mathrm{ml} /$ $\min / 1.73 \mathrm{~m}^{2}(\mathrm{p}=0.025)$, showed a rapid decline in renal function (fig. 3 ).

\section{Performance of uPOSTN/Cr in Predicting ESRD}

The ROC analysis of uPOSTN ( $\mathrm{pg} / \mathrm{mgCr}$ ) in predicting ESRD in $\operatorname{IgAN}$ is illustrated in figure 4. Urinary POSTN/Cr $>1,354.5 \mathrm{pg} / \mathrm{mgCr}$ demonstrates a $74.1 \%$ specificity and $73.7 \%$ sensitivity for predicting ESRD occurrence. The performance of uPOSTN/Cr at the time of renal biopsy in predicting ESRD is also demonstrated in figure 4 . In the time-dependent ROC curve, the AUC values for ESRD at 12, 24 and 36 months were increased over time to $0.625,0.772$ and 0.77 , respectively (online suppl. fig. S3). There was only a weak effect of incremental increases in uPOSTN/Cr level on serum creatinine and uPCr levels. Even though uPCr (especially more than $1 \mathrm{~g} /$ day) is a well-known risk factor for poor renal outcome, the ROC did not exhibit a significant improvement in predictive value over serum creatinine levels (online suppl. table S2).

\section{Tissue Periostin and $\mathrm{uPOSTN} / \mathrm{Cr}$}

Among the 345 enrolled patients, only 75 unstained slides were available and used for conducting periostin immunohistochemistry. The correlation between tissue periostin expression and uPOSTN/Cr is demonstrated in figure 5. Periostin highly stained in patients with 
Fig. 2. Renal outcome evaluation. KaplanMeier curve (a) for the occurrence of ESRD (log-rank $\mathrm{p}=0.003$ ), (b) for ESRD and/or eGFR decrease $>30 \%(\log$-rank $\mathrm{p}=0.033)$ and (c) for the ESRD and/or eGFR decrease $>50 \%$ (log-rank $\mathrm{p}=0.046$ ) showed significant differences between group $1+2$ and group 3. The patients with (d) ESRD $(\mathrm{p}<0.001),(\mathbf{e})$ eGFR decrease $>30 \%(\mathrm{p}=$ $0.002)$ and (f) eGFR decrease $>50 \%(\mathrm{p}=$ 0.046 ) showed higher ln-uPOSTN/Cr levels than patients without renal outcome.

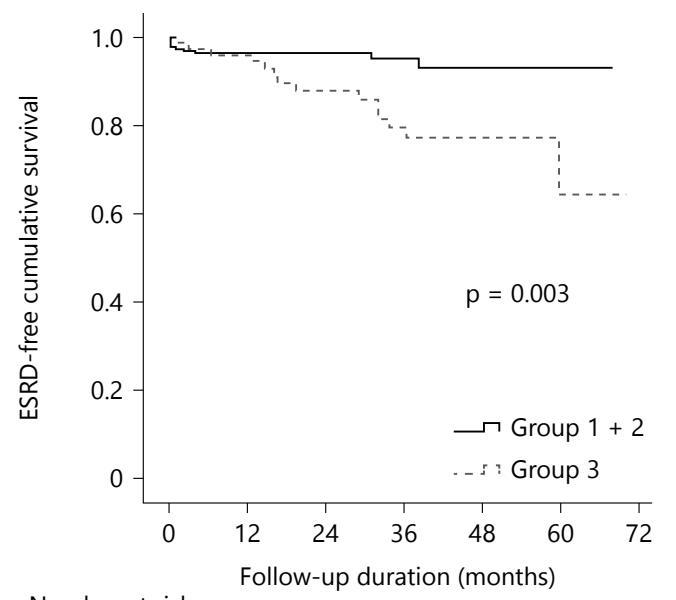

Number at risk

Group $1+2 \quad 155 \quad 108 \quad 45 \quad 20 \quad 4$

$\begin{array}{lllllll}\text { a } & \text { Group } 3 & 63 & 48 & 34 & 20 & 4\end{array}$

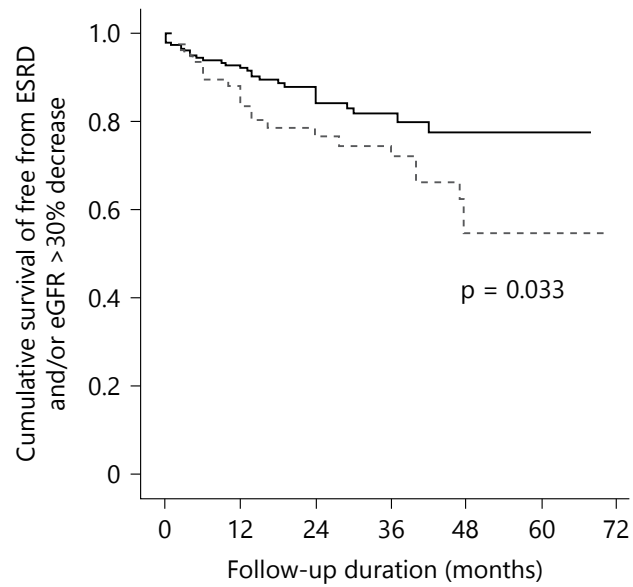

Number at risk

$\begin{array}{llllll}\text { Group } 1+2 & 149 & 94 & 41 & 18 & 3\end{array}$

b $\quad \begin{array}{lllllll}\text { Group } 3 & 58 & 41 & 31 & 14 & 3\end{array}$

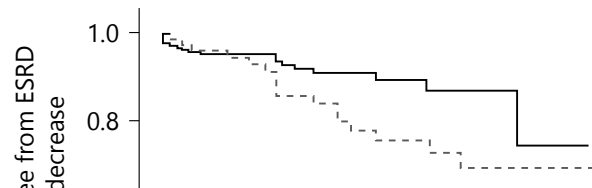

常

$\frac{4}{5}$

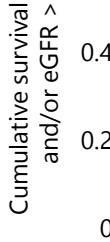

0.6

0.4

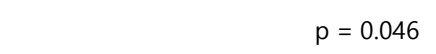

0.2

$-$

Number at risk

$\begin{array}{rccccc}\text { Group 1+2 } & 151 & 97 & 43 & 19 & 3 \\ \text { Group 3 } & 62 & 46 & 33 & 19 & 4\end{array}$

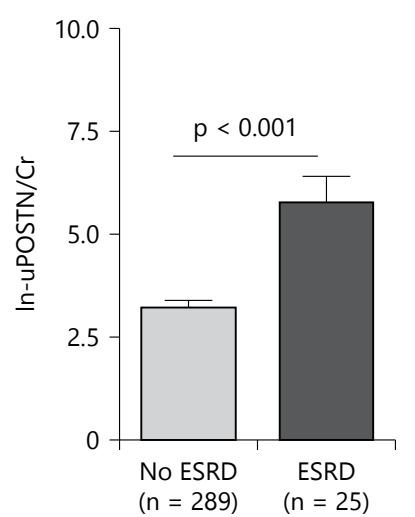

d
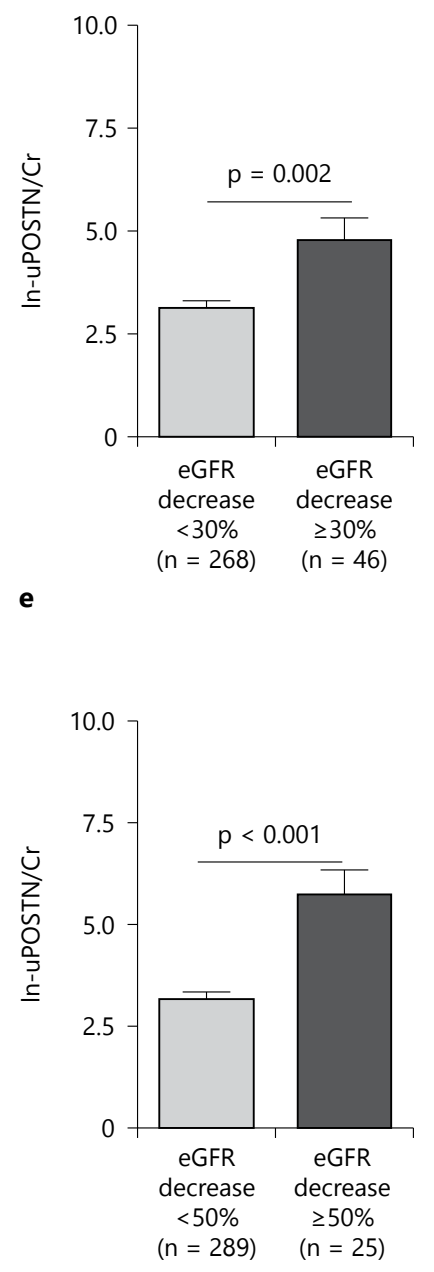
Fig. 3. Comparison of $\Delta \mathrm{eGFR} /$ year in the 2 groups (subcategorized by initial eGFR). We compared annual eGFR decrease $(\triangle \mathrm{eGFR} /$ year) only in patients who were followed up for more than 1 year. The patients in group 3 showed a rapid decline in renal function both whose initial eGFR (a) $\geq 60 \mathrm{ml} / \mathrm{min} / 1.73 \mathrm{~m}^{2}(\mathrm{p}=0.043)$ and $(\mathbf{b})$ $<60 \mathrm{ml} / \mathrm{min} / 1.73 \mathrm{~m}^{2}(\mathrm{p}=0.025)$.

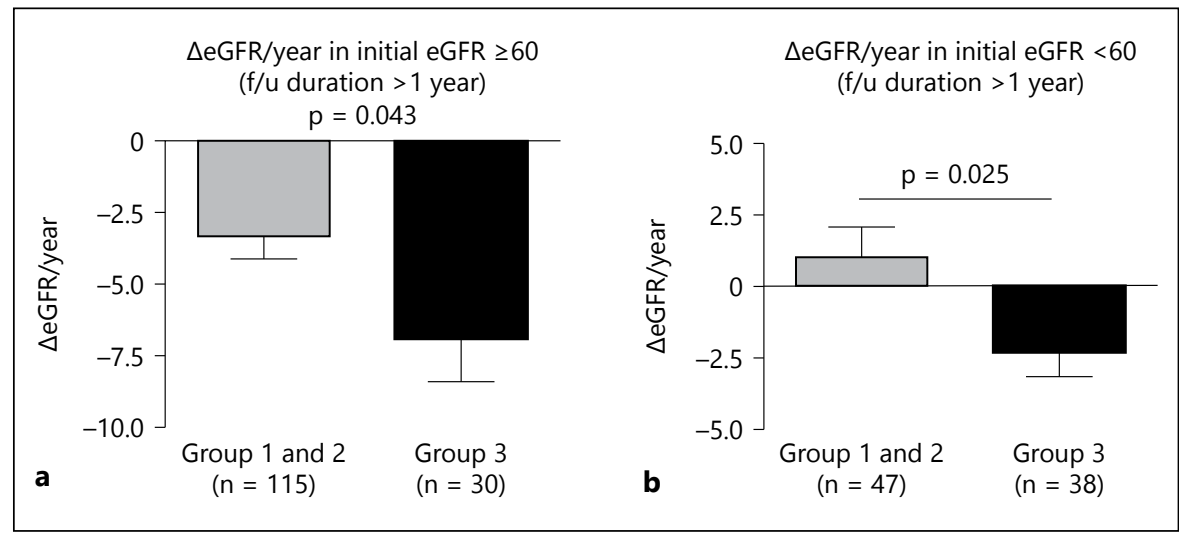

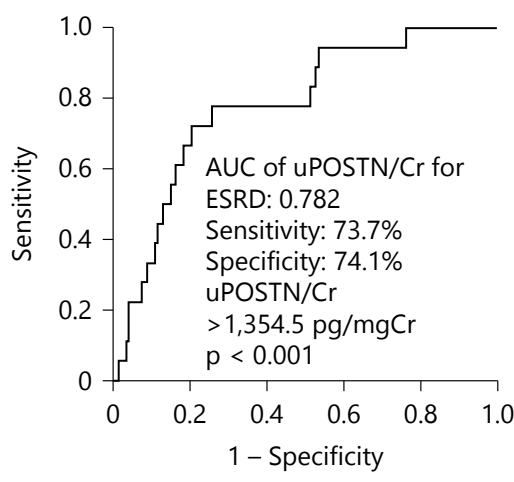

Fig. 4. ROC analysis and performance in predicting ESRD. Urinary POSTN/Cr $>1,354.5 \mathrm{pg} / \mathrm{mgCr}$ demonstrates $74.1 \%$ specificity and $73.7 \%$ sensitivity in predicting ESRD occurrence. The performance of uPOSTN/Cr at the time of renal biopsy in predicting ESRD is also demonstrated.

Table 2. Multivariate analysis for the occurrence of ESRD

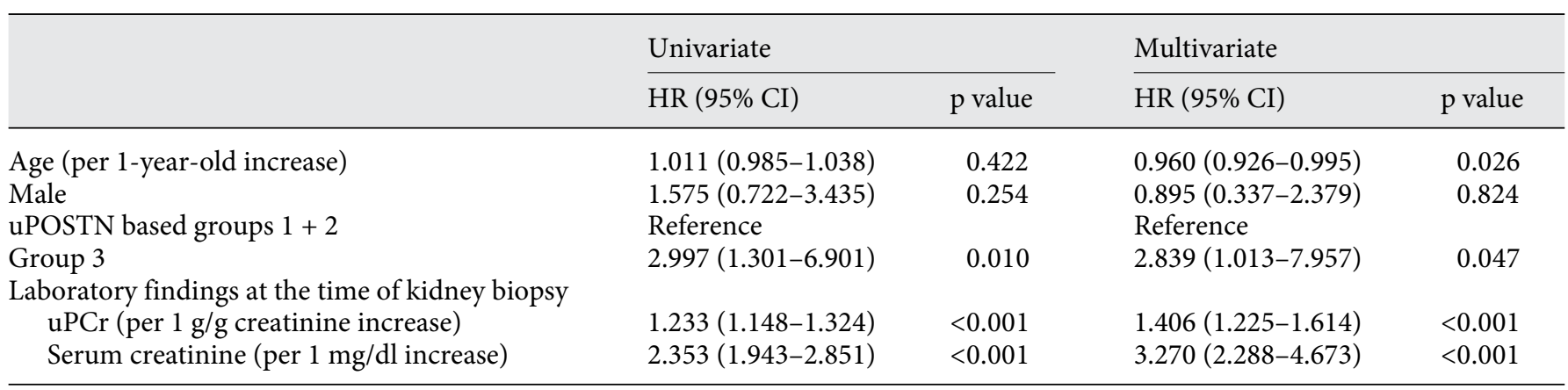

high uPOSTN/Cr level (fig. 5a). In morphometry analysis for periostin IHC slides, patients with a high uPOSTN/Cr level showed higher tissue periostin expression (fig. 5b). There was a positive correlation between uPOSTN/Cr and tissue POSTN $(r=0.292 ; \mathrm{p}=$
0.011; fig. 5c). On survival analysis for ESRD occurrence by morphometry result-based grouping, ESRD occurred only in patients with high tissue periostin expression ( $p=0.003$; fig. $5 \mathrm{~d})$. 


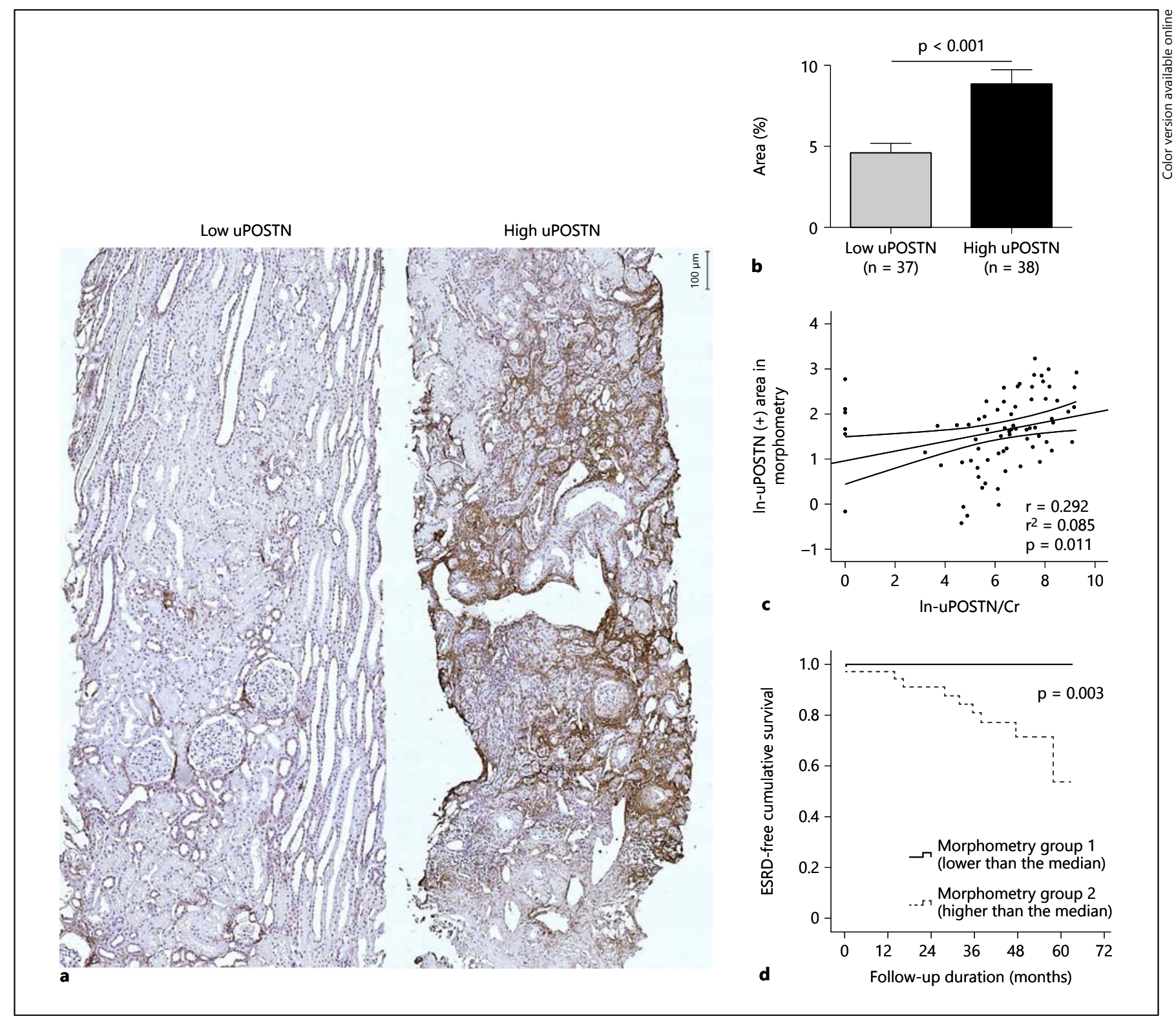

Fig. 5. Tissue periostin expression and uPOSTN in IgAN patients. a Periostin immunohistochemistry in IgAN patients (original magnification: $\times 50$ ). b Morphometry results for periostin positive area $(\%)$ in uPOSTN 2 groups $(\mathrm{p}<0.001)$. c Correlation between $\mathrm{ln}$-uPOSTN/Cr and morphometry result ( $\mathrm{r}=0.292$;

\section{Periostin-Induced Collagen Ia1 Expression in Cultured Collecting Duct Cells}

The $\mathrm{H} 5$ cells were treated with $10 \mathrm{ng}$ TGF- $\beta$ (positive control) or $0.25,0.5$ or $1.0 \mu \mathrm{g}$ periostin for $24 \mathrm{~h}$. As shown in online supplementary figure S4, periostin treatment increased collagen Ial and fibronectin expression in a dose-dependent manner (online suppl. fig. S4).

uPOSTN/Cr and Renal Outcome in IgAN $\mathrm{p}=0.011)$. d Kaplan-Meier curve for the occurrence of ESRD according to morphometry positivity area (\%)-based grouping (lower than the median value vs. high than the median value). The ESRD occurred only in the patients with high tissue periostin expression (log-rank, $\mathrm{p}=0.003)$.

\section{Discussion}

This study shows that a higher uPOSTN/Cr level at renal biopsy is associated with a greater decline in eGFR during follow-up and occurrence of ESRD. Pathologic findings such as IF/TA, interstitial inflammation and hyaline arteriolosclerosis were also related with a high 
uPOSTN/Cr level. Tissue periostin expression was correlated not only with uPOSTN/Cr but also with the renal outcome. In addition, a brief in vitro experiment showed that periostin induced the representative fibrosis marker in a dose-dependent manner. This association was observed in patients with conserved renal function (eGFR $>60 \mathrm{ml} / \mathrm{min} / 1.73 \mathrm{~m}^{2}$ ). Taken together, periostin is a possible early-stage marker of renal fibrosis and a promising urinary biomarker for evaluation of renal injury and prediction of outcome in IgAN.

Evidence for periostin as a biomarker in specific renal disease is now emerging [14, 15, 17-19]. Periostin is known to induce fibrosis by a dual mechanism: increased inflammatory reaction and upregulated TGF- $\beta$ signaling [20]. Periostin itself is produced by fibrosis induction, and it promotes extracellular matrix deposition, mesenchymal cell proliferation and wound closure by stimulating the phosphorylation of FAK, p38 and ERK 42/44 [20, $21]$. However, no previous reports on the association of periostin and IgAN exist, although IgAN is the most common glomerulonephritis worldwide. Our results showing a relationship between higher uPOSTN/Cr levels and severe renal fibrosis and poor renal outcomes in IgAN are consistent with those from previous studies on other renal diseases.

uPOSTN/Cr level was correlated with plasma periostin, but plasma periostin failed to show significant correlations with various other parameters. With regard to renal disease, renal periostin expression has been reported in both glomerulosclerosis and renal tubular fibrosis. Given its nature, serum periostin levels can be affected by systemic conditions in many organs or tissues. However, uPOSTN level can be directly affected by local renal tissue periostin expression, especially through tubular secretion. Although we adjusted uPOSTN by urine creatinine level in this study because we considered that every urine sample was diluted differently, only 4 patients were classified into a different group when we used direct uPOSTN level without creatinine adjustment. This finding also shows the possibility that most of the periostin in the urine comes from the tubules. Moreover, uPOSTN/Cr level was well correlated with tissue periostin expression, and tissue periostin expression affected renal outcome. It is difficult to draw a definitive conclusion, as uPOSTN might be mainly pulled by tubular secretion than glomerular filtration.

In IgAN-related research, ESRD is a difficult renal outcome to study because it takes a long time from diagnosis to onset. However, this study showed a significant difference in the occurrence of ESRD between the low- er uPOSTN level group (group $1+2$ ) and the higher uPOSTN level group (group 3) during the 2.25 years of mean follow-up. This may imply that poor renal outcome is related to baseline characteristics, such as eGFR at the time of renal biopsy. Annual loss of eGFR $(\Delta \mathrm{eGFR} /$ year) in patients with IgAN is known to vary from -8.4 to $32.3 \mathrm{ml} / \mathrm{min} / 1.73 \mathrm{~m}^{2}[22,23]$, and the proportion of progression to ESRD is between 6 and $43 \%$ over 10 years. Periostin is associated with renal fibrosis, and it would be naturally associated with a decrease in eGFR. To overcome the different follow-up duration and significantly different baseline eGFR between the groups, we divided the patients by initial eGFR $\left(60 \mathrm{ml} / \mathrm{min} / 1.73 \mathrm{~m}^{2}\right)$ and then compared $\triangle \mathrm{eGFR} /$ year between the groups to express and assess the independent effect of periostin on renal outcomes. In this analysis, the commonly high uPOSTN/ $\mathrm{Cr}$ population had a significantly high $\triangle \mathrm{eGFR} / \mathrm{year}$. Moreover, when we adjusted for age, sex, uPCr and serum creatinine at the time of renal biopsy, categorization into group 3 was independently associated with ESRD occurrence.

Periostin showed moderate utility as a biomarker in ROC analysis. However, there was the obvious limitation that we could not perform quantitative analysis in all cases because the uPOSTN level was undetectable in 148 urine samples by the ELISA kit even on repetitive measurements. We evaluated the predictive performance of uPOSTN/Cr at the time of renal biopsy using time-dependent ROC curves at 1,2 and 3 years after diagnosis, which showed gradually increasing predictability. In patients who developed ESRD within a short duration (within 1 or 2 years from diagnosis), there could be other factors (such as low baseline eGFR) that strongly effected ESRD occurrence than uPOSTN/Cr levels.

Because uPOSTN/Cr levels were correlated with tissue periostin expression in various analyses and plasma periostin levels were not correlated with tissue findings, there is a high probability that uPOSTN was excreted from local kidney tissue and reflects the tissue periostin concentration. These findings, together with those from previous studies, reported a correlation between tissue periostin expression and renal fibrosis (especially IF/TA); uPOSTN measurement by ELISA is a useful tool to detect tissue periostin expression and predict renal outcome. A brief in vitro experiment showed increase in collagen Ia1 mRNA expression in a dose-dependent manner when recombinant periostin was administered to $\mathrm{H} 5$ cells. This result implies that periostin not only represents a simple biomarker for fibrosis, but also induces fibrosis as an ac- 
tive matricellular protein. This finding was also suggested in previous study [20].

Finding urinary biomarkers for specific disease entities is useful and has various advantages such as convenient sample collection, high specificity to local renal expression and non-invasiveness. Until now, the diagnosis of IgAN is based on histological confirmation; hence, urinary biomarkers cannot replace kidney biopsy. There have been many classifications and pathologic staging as efforts to predict the prognosis of IgAN, and the efforts are continued. In this study, uPOSTN was highly related to renal fibrosis; a high uPOSTN level reflected high tissue POSTN expression and correlated with poor renal outcome.

The strength of this study is that all the urine, plasma and tissue samples were collected at the same time and diagnostic point in each individual disease course. Moreover, as all the patients in this study were included in prospective cohort, researchers were able to collect patients' serial follow-up data after diagnosis.

The major limitation of this study is that the ELISA kit was not sensitive enough and the uPOSTN level was too low to be detected. There were 148 patients with an undetectable POSTN level, who were categorized separately into group 1 . This made the patients with undetectable uPOSTN level to be excluded in the quantitative analysis such as ROC and this might have caused selection bias. Even though there have been at least 100 articles published about serum POSTN, there are only limited reports about 'urine' POSTN. This may be due to the difficulty in detecting uPOSTN using commercially available ELISA kits. In addition, most patients in this study underwent kidney biopsy and were enrolled between 2009 and 2012; therefore, MEST criteria in the Oxford classification could not be applied in the pathologic analysis although we recognize that it offers precise prediction and risk stratification [24-26]. However, the IF/TA scoring system used in this study was similar to the MEST criteria, with the same T score, and only endocapillary hypercellularity from the MEST criteria was not included in this study. In the VALIGA study, endocapillary hypercellularity did not predict poor renal outcomes. Furthermore, in the effect sizes of pathology findings using the MEST criteria, the T score showed the greatest hazards ratio [25]. Furthermore, due to the cross-sectional design of the relationship between uPOSTN/Cr levels and pathologic findings, only associations can be inferred.

In summary, the progression of CKD to ESRD is commonly related to renal fibrosis. This study estab- lished a clear relation between high uPOSTN/Cr levels and renal fibrosis, high tissue periostin expression, low eGFR and rapid decline in renal function in patients with IgAN, the most common glomerulonephritis worldwide. Periostin is a possible early-stage marker for renal fibrosis and a promising urinary biomarker for evaluation of renal fibrosis and prediction of renal outcome in IgAN.

\section{Acknowledgments}

The biospecimens for this study were provided by the Seoul National University Hospital Human Biobank and the Seoul National University Boramae Medical Center Human Biobank under the Nephrology Department. Seoul National University Hospital Human Biobank is a member of the National Biobank of Korea, which is supported by the Ministry of Health and Welfare.

The authors deeply thank Remi Piedagnel at INSERM laboratory (French Institute of Health and Medical Research, Paris, France) for his kind offer of $\mathrm{H} 5$ cells and the cell culture protocol. We also thank Bancha Satirapoj, nephrologist at Phramongkutklao Hospital, Bangkok, Thailand, for sharing his experience with uPOSTN analysis using ELISA.

\section{Funding}

This study was supported by research grant from Pfizer Inc., N.Y., USA.

\section{Disclosure Statement}

The authors report no conflicts of interest regarding this publication.

References

1 Liu Y: Renal fibrosis: new insights into the pathogenesis and therapeutics. Kidney Int 2006;69:213-217.

2 Wyatt RJ, Julian BA: IgA nephropathy. N Engl J Med 2013;368:2402-2414.

3 Chang JH, Kim DK, Kim HW, et al: Changing prevalence of glomerular diseases in Korean adults: a review of 20 years of experience. Nephrol Dial Transplant 2009;24: 2406-2410.

4 Layton JB, Hogan SL, Jennette CE, et al: Discrepancy between medical evidence form 2728 and renal biopsy for glomerular diseases. Clin J Am Soc Nephrol 2010;5:2046-2052.

5 Lee H, Kim DK, Oh KH, et al: Mortality of IgA nephropathy patients: a single center experience over 30 years. PLoS One 2012; 7:e51225. 
6 Abdalla MA, Haj-Ahmad Y: Promising urinary protein biomarkers for the early detection of hepatocellular carcinoma among high-risk hepatitis $\mathrm{C}$ virus egyptian patients. J Cancer 2012;3:390-403.

7 Conway SJ, Izuhara K, Kudo Y, et al: The role of periostin in tissue remodeling across health and disease. Cell Mol Life Sci 2014;71:1279-1288.

8 Braun N, Sen K, Alscher MD, et al: Periostin: a matricellular protein involved in peritoneal injury during peritoneal dialysis. Perit Dial Int 2013;33:515-528.

9 Zhao S, Wu H, Xia W, et al: Periostin expression is upregulated and associated with myocardial fibrosis in human failing hearts. J Cardiol 2014;63:373-378.

10 Katsuragi N, Morishita R, Nakamura N, et al: Periostin as a novel factor responsible for ventricular dilation. Circulation 2004;110:18061813.

11 Ling L, Cheng Y, Ding L, Yang X: Association of serum periostin with cardiac function and short-term prognosis in acute myocardial infarction patients. PLoS One 2014; 9:e88755.

12 Kudo Y, Siriwardena BS, Hatano H, Ogawa I, Takata T: Periostin: novel diagnostic and therapeutic target for cancer. Histol Histopathol 2007;22:1167-1174.

13 Ito T, Suzuki A, Imai E, et al: Tornado extraction: a method to enrich and purify RNA from the nephrogenic zone of the neonatal rat kidney. Kidney Int 2002;62:763-769.

14 Satirapoj B, Wang Y, Chamberlin MP, et al: Periostin: novel tissue and urinary biomarker of progressive renal injury induces a coordinated mesenchymal phenotype in tubular cells. Nephrol Dial Transplant 2012;27:2702-2711.

15 Satirapoj B, Witoon R, Ruangkanchanasetr P, et al: Urine periostin as a biomarker of renal injury in chronic allograft nephropathy. Transplant Proc 2014;46:135-140.

16 Sen K, Lindenmeyer MT, Gaspert A, et al: Periostin is induced in glomerular injury and expressed de novo in interstitial renal fibrosis. Am J Pathol 2011;179:1756-1767.

17 Wallace DP, Quante MT, Reif GA, et al: Periostin induces proliferation of human autosomal dominant polycystic kidney cells through alphaV-integrin receptor. Am J Physiol Renal Physiol 2008;295:F1463-F1471.

18 Satirapoj B, Tassanasorn S, Charoenpitakchai $\mathrm{M}$, Supasyndh O: Periostin as a tissue and urinary biomarker of renal injury in type 2 diabetes mellitus. PLoS One 2015;10:e0124055.

19 Wantanasiri P, Satirapoj B, Charoenpitakchai M, Aramwit P: Periostin: a novel tissue biomarker correlates with chronicity index and renal function in lupus nephritis patients. Lupus 2015;24:835-845.

20 Mael-Ainin M, Abed A, Conway SJ, Dussaule JC, Chatziantoniou C: Inhibition of periostin expression protects against the development of renal inflammation and fibrosis. J Am Soc Nephrol 2014;25:1724-1736.

21 Naik PK, Bozyk PD, Bentley JK, et al: Periostin promotes fibrosis and predicts progression in patients with idiopathic pulmonary fibrosis. Am J Physiol Lung Cell Mol Physiol 2012;303:L1046-L1056.

22 Shi SF, Wang SX, Jiang L, et al: Pathologic predictors of renal outcome and therapeutic efficacy in IgA nephropathy: validation of the oxford classification. Clin J Am Soc Nephrol 2011;6:2175-2184.

23 Lundberg S, Qureshi AR, Olivecrona S, et al: FGF23, albuminuria, and disease progression in patients with chronic IgA nephropathy. Clin J Am Soc Nephrol 2012;7:727-734.

24 Working Group of the International IgA Nephropathy Network and the Renal Pathology Society, Roberts IS, et al: The Oxford classification of IgA nephropathy: pathology definitions, correlations, and reproducibility. Kidney Int 2009;76:546-556.

25 Coppo R, Troyanov S, Bellur S, et al: Validation of the Oxford classification of IgA nephropathy in cohorts with different presentations and treatments. Kidney Int 2014;86:828-836.

26 Barbour SJ, Espino-Hernandez G, Reich HN, et al: The MEST score provides earlier risk prediction in IgA nephropathy. Kidney Int 2015, Epub ahead of print. 